\title{
Erratum to: Thermodynamic Modeling of the Fe-Mn-C and the Fe-Mn-Al Systems Using the Modified Quasichemical Model for Liquid Phase
}

Min-Su Kim and Youn-Bae Kang

Erratum to: JPEDAV (2015) 36:453-470

DOI 10.1007/s11669-015-0401-7

The authors regret the following typing errors in Tables 3 and 4 , the correct tables are (corrected part is seen with arrow $(\rightarrow)$ ): 
Table 3 Summary of model parameters in the Fe-Mn-C system ( $/ / \mathrm{mol})$

\begin{tabular}{|c|c|c|}
\hline Phase & Model & Parameter \\
\hline Liquid & $(\mathrm{Fe}, \mathrm{Mn}, \mathrm{C})$ & $\begin{array}{l}Z_{\mathrm{FeFe}}^{\mathrm{Fe}}=Z_{\mathrm{MnMn}}^{\mathrm{Mn}}=Z_{\mathrm{CC}}^{\mathrm{C}}=Z_{\mathrm{FeMn}}^{\mathrm{Fe}}=Z_{\mathrm{FeMn}}^{\mathrm{Mn}}=Z_{\mathrm{FeC}}^{\mathrm{C}}=Z_{\mathrm{MnC}}^{\mathrm{C}}=6, Z_{\mathrm{FeC}}^{\mathrm{Fe}}=Z_{\mathrm{MnC}}^{\mathrm{Mn}}=3 \\
\Delta g_{\mathrm{FeC}}=-30459.5+3.14 T-1129.7 X_{\mathrm{FeFe}}^{\left[{ }^{[4]}\right.} \\
\Delta g_{\mathrm{FeMn}}=-1338.9+0.17 T-334.7 X_{\mathrm{MnMn}}+418.4 X_{\mathrm{FeFe}}{ }^{[9]} \\
\Delta g_{\mathrm{MnC}}=-42677+4.50 T+(2092+1.75 T) X_{\mathrm{MnMn}}{ }^{[5]} \\
\Delta g_{\mathrm{FeMn}(\mathrm{C})}=-8368+9623 X_{\mathrm{MnMn}} \\
\text { "Toop-like" interpolation }(\mathrm{C} \text { as the asymmetric component })^{[52]}\end{array}$ \\
\hline fcc & $(\mathrm{Fe}, \mathrm{Mn})_{1}(\mathrm{C}, \mathrm{Va})_{1}$ & $\begin{array}{l}G_{\mathrm{Fe}: \mathrm{Va}}=\mathrm{GFCCFE}, G_{\mathrm{Mn}: \mathrm{Va}}=\mathrm{GFCCMN} \\
G_{\mathrm{Fe}: \mathrm{C}}=\mathrm{GFCCFE}+\mathrm{GHSERCC}+77207-15.88 T^{[23]} \\
G_{\mathrm{Mn}: \mathrm{C}}=\mathrm{GHSERMN}+\mathrm{GHSERCC}+13.66 T^{[30]} \\
L_{\mathrm{Fe}, \mathrm{Mn}: \mathrm{Va}}=-7762+3.87 T-259\left(y_{\mathrm{Fe}}^{\prime}-y_{\mathrm{Mn}}^{\prime}\right)^{[27]} \\
L_{\mathrm{Fe}: \mathrm{C}, \mathrm{Va}}=-34671^{[23]} \\
(\rightarrow) L_{\mathrm{Mn}: \mathrm{C}, \mathrm{Va}}=-21388.6+23012\left(y_{\mathrm{C}}^{\prime \prime}-y_{\mathrm{Va}}^{\prime \prime}\right)^{[5]} \\
L_{\mathrm{Fe}, \mathrm{Mn}: \mathrm{C}}=-836.8 \\
T_{\mathrm{cFe}: \mathrm{Va}}=-201, T_{\mathrm{cMn}: \mathrm{Va}}=-1620, \beta_{\mathrm{Fe}: \mathrm{Va}}=-2.1, \beta_{\mathrm{Mn}: \mathrm{Va}}=-1.86^{[27]} \\
T_{\mathrm{cFe}, \mathrm{Mn}: \mathrm{Va}}=-2282-2068\left(y_{\mathrm{Fe}}^{\prime}-y_{\mathrm{Mn}}^{\prime}\right)^{[27]}\end{array}$ \\
\hline bcc_A2 & $(\mathrm{Fe}, \mathrm{Mn})_{1}(\mathrm{C}, \mathrm{Va})_{3}$ & $\begin{array}{l}G_{\mathrm{Fe}: \mathrm{Va}}=\text { GHSERFE, } G_{\mathrm{Mn}: \mathrm{Va}}=\mathrm{GBCCMN} \\
G_{\mathrm{Fe}: \mathrm{C}}=\mathrm{GHSERFE}+3 \mathrm{GHSERCC}+322054+75.67 T^{[23]} \\
G_{\mathrm{Mn}: \mathrm{C}}=\mathrm{GHSERMN}+3 \mathrm{GHSERCC}+10000+30 T^{[30]} \\
L_{\mathrm{Fe}, \mathrm{Mn}: \mathrm{Va}}=-2759+1.24 T^{[27]} \\
L_{\mathrm{Fe}: \mathrm{C}, \mathrm{Va}}=-190 T^{[23]} \\
T_{\mathrm{cFe}: \mathrm{Va}}=1043, T_{\mathrm{cMn}: \mathrm{Va}}=-580, \beta_{\mathrm{Fe}: \mathrm{Va}}=2.22, \beta_{\mathrm{Mn}: \mathrm{Va}}=-0.27^{[27]} \\
T_{\mathrm{cFe}, \mathrm{Mn}: \mathrm{Va}}=123^{[27]}\end{array}$ \\
\hline cbcc & $(\mathrm{Fe}, \mathrm{Mn})_{1}(\mathrm{C}, \mathrm{Va})_{1}$ & $\begin{array}{l}G_{\mathrm{Fe}: \mathrm{Va}}=\mathrm{GHSERFE}+4745,{ }^{[27]} G_{\mathrm{Mn}: \mathrm{Va}}=\mathrm{GHSERMN} \\
G_{\mathrm{Fe}: \mathrm{C}}=\mathrm{GHSERFE}+\mathrm{GHSERCC}+50000 \\
G_{\mathrm{Mn}: \mathrm{C}}=\mathrm{GHSERMN}+\mathrm{GHSERCC}+5000+22.47 T^{[30]} \\
L_{\mathrm{Fe}, \mathrm{Mn}: \mathrm{Va}}=-10184^{[27]} \\
L_{\mathrm{Fe}: \mathrm{C}, \mathrm{Va}}=-34671^{[18]} \\
L_{\mathrm{Mn}: \mathrm{C}, \mathrm{Va}}=-50208^{[5]} \\
(\rightarrow) T_{\mathrm{cMn}: \mathrm{Va}}=-285, \beta_{\mathrm{Mn}: \mathrm{Va}}=-0.66^{[29]}\end{array}$ \\
\hline cub & $(\mathrm{Fe}, \mathrm{Mn})_{1}(\mathrm{C}, \mathrm{Va})_{1}$ & $\begin{array}{l}G_{\mathrm{Fe}: \mathrm{Va}}=\mathrm{GHSERFE}+3745,{ }^{[27]} G_{\mathrm{Mn}: \mathrm{Va}}=\mathrm{GCUBMN} \\
G_{\mathrm{Fe}: \mathrm{C}}=\mathrm{GHSERFE}+\mathrm{GHSERCC}+90000^{[18]} \\
G_{\mathrm{Mn}: \mathrm{C}}=\mathrm{GHSERMN}+\mathrm{GHSERCC}+2607^{[30]} \\
L_{\mathrm{Fe}, \mathrm{Mn}: \mathrm{Va}}=-11518+2.82 T^{[27]} \\
L_{\mathrm{Fe}: \mathrm{C}, \mathrm{Va}}=-34671^{[18]} \\
L_{\mathrm{Mn}: \mathrm{C}, \mathrm{Va}}=-10460^{[5]}\end{array}$ \\
\hline hep & $(\mathrm{Fe}, \mathrm{Mn})_{1}(\mathrm{C}, \mathrm{Va})_{0.5}$ & $\begin{array}{l}G_{\mathrm{Fe}: \mathrm{Va}}=\mathrm{GHCPFE}, G_{\mathrm{Mn}: \mathrm{Va}}=\mathrm{GHCPMN} \\
G_{\mathrm{Fe}: \mathrm{C}}=\mathrm{GFCCFE}+0.5 \mathrm{GHSERCC}+48512.5-8.78 T \\
G_{\mathrm{Mn}: \mathrm{C}}=\mathrm{GHSERMN}+0.5 \mathrm{GHSERCC}-9000-1.07 T^{[30]} \\
L_{\mathrm{Fe}, \mathrm{Mn}: \mathrm{Va}}=-5748+3.87 T+273\left(y_{\mathrm{Fe}}^{\prime}-y_{\mathrm{Mn}}^{\prime}\right)^{[20]} \\
L_{\mathrm{Fe}: \mathrm{C}, \mathrm{Va}}=-17335^{[20]} \\
L_{\mathrm{Mn}: \mathrm{C}, \mathrm{Va}}=-2092-3.14 T-5230\left(y_{\mathrm{C}}^{\prime \prime}-y_{\mathrm{Va}}^{\prime \prime}\right)^{[5]} \\
L_{\mathrm{Fe}, \mathrm{Mn}: \mathrm{C}}=18828-47.36 T-33472\left(y_{\mathrm{Fe}}^{\prime}-y_{\mathrm{Mn}}^{\prime}\right) \\
(\rightarrow) T_{\mathrm{cMn}: \mathrm{Va}}=-1620, \beta_{\mathrm{Mn}: \mathrm{Va}}=-1.86^{[27]}\end{array}$ \\
\hline $\mathrm{M}_{3} \mathrm{C}$ & $(\mathrm{Fe}, \mathrm{Mn})_{3}(\mathrm{C})_{1}$ & $\begin{array}{l}G_{\mathrm{Fe}: \mathrm{C}}=\mathrm{GCEMFE},{ }^{[25]} G_{\mathrm{Mn}: \mathrm{C}}=3 \mathrm{GHSERMN}+\mathrm{GHSERCC}-32000-4.1 T^{[5]} \\
L_{\mathrm{Fe}, \mathrm{Mn}: \mathrm{C}}=-3347.2-2510.4\left(y_{\mathrm{Fe}}^{\prime}-y_{\mathrm{Mn}}^{\prime}\right) \\
(\rightarrow) T_{\mathrm{cFe}: \mathrm{C}}=485, \beta_{\mathrm{Fe}: \mathrm{C}}=1.008^{[25]}\end{array}$ \\
\hline $\mathrm{M}_{5} \mathrm{C}_{2}$ & $(\mathrm{Fe}, \mathrm{Mn})_{5}(\mathrm{C})_{2}$ & $\begin{array}{l}G_{\mathrm{Fe}: \mathrm{C}}=\frac{5}{3} \mathrm{GCEMFE}+\frac{1}{3} \mathrm{GHSERCC}+14100-7.35 T \\
G_{\mathrm{Mn}: \mathrm{C}}=5 \mathrm{GHSERMN}+2 \mathrm{GHSERCC}-62302-5 T^{[5]} \\
L_{\mathrm{Fe}, \mathrm{Mn}: \mathrm{C}}=-5020.8\end{array}$ \\
\hline $\mathrm{M}_{7} \mathrm{C}_{3}$ & $(\mathrm{Fe}, \mathrm{Mn})_{7}(\mathrm{C})_{3}$ & $\begin{array}{l}G_{\mathrm{Fe}: \mathrm{C}}=\frac{7}{3} \mathrm{GCEMFE}+\frac{2}{3} \mathrm{GHSERCC}+30000-15.45 T \\
G_{\mathrm{Mn}: \mathrm{C}}=7 \mathrm{GHSERMN}+3 \mathrm{GHSERCC}-91002-6.2 T^{[5]} \\
L_{\mathrm{Fe}, \mathrm{Mn}: \mathrm{C}}=-7893.53+6.46 T\end{array}$ \\
\hline $\mathrm{M}_{23} \mathrm{C}_{6}$ & $(\mathrm{Fe}, \mathrm{Mn})_{20}(\mathrm{Fe}, \mathrm{Mn})_{3}(\mathrm{C})_{6}$ & $\begin{array}{l}G_{\mathrm{Fe}: \mathrm{Fe}: \mathrm{C}}=\frac{23}{3} \mathrm{GCEMFE}-\frac{5}{3} \mathrm{GHSERCC}+15000 \\
G_{\mathrm{Mn}: \mathrm{Mn}: \mathrm{C}}=23 \mathrm{GHSERMN}+6 \mathrm{GHSERCC}-245501-2.44 T^{[5]} \\
23 G_{\mathrm{Fe}: \mathrm{Mn}: \mathrm{C}}=20 G_{\mathrm{Fe}: \mathrm{Fe}: \mathrm{C}}+3 G_{\mathrm{Mn}: \mathrm{Mn}: \mathrm{C}}, 23 G_{\mathrm{Mn}: \mathrm{Fe}: \mathrm{C}}=20 G_{\mathrm{Mn}: \mathrm{Mn}: \mathrm{C}}+3 G_{\mathrm{Fe}: \mathrm{Fe}: \mathrm{C}}^{[20]} \\
L_{\mathrm{Fe}, \mathrm{Mn}: \mathrm{Fe}, \mathrm{Mn}: \mathrm{C}}=-79496\end{array}$ \\
\hline
\end{tabular}


Table 4 Summary of model parameters in the Fe-Mn-Al system (J/mol)

\begin{tabular}{|c|c|c|}
\hline Phase & Model & Parameter \\
\hline Liquid & $(\mathrm{Fe}, \mathrm{Mn}, \mathrm{Al})$ & $\begin{array}{l}Z_{\mathrm{FeFe}}^{\mathrm{Fe}}=Z_{\mathrm{MnMn}}^{\mathrm{Mn}}=Z_{\mathrm{AlAl}}^{\mathrm{Al}}=Z_{\mathrm{FeMn}}^{\mathrm{Fe}}=Z_{\mathrm{FeMn}}^{\mathrm{Mn}}=Z_{\mathrm{FeAl}}^{\mathrm{Fe}}=Z_{\mathrm{FeAl}}^{\mathrm{Al}}=Z_{\mathrm{AlMn}}^{\mathrm{Al}}=Z_{\mathrm{AlMn}}^{\mathrm{Mn}}=6 \\
\Delta g_{\mathrm{FeAl}}=-20292.4+3.35 T+(-1673.6-1.26 T) X_{\mathrm{FeFe}}+(-10460+4.18 T) X_{\mathrm{AlAl}}-1046 X_{\mathrm{FeFe}}^{2}{ }^{[10]} \\
\Delta g_{\mathrm{FeMn}}=-1338.9+0.167 T-334.7 X_{\mathrm{MnMn}}+418.4 X_{\mathrm{FeFe}}{ }^{[9]} \\
\Delta g_{\mathrm{AlMn}}=-17572.8+6.07 T+(-4602.4+1.26 T) X_{\mathrm{AlAl}}^{2}+(-4184+1.67 T) X_{\mathrm{MnMn}}^{2} \\
\text { "Toop-like" interpolation (Al as the asymmetric component })^{[52]}\end{array}$ \\
\hline fcc & $(\mathrm{Fe}, \mathrm{Mn}, \mathrm{Al})_{1}(\mathrm{Va})_{1}$ & $\begin{array}{l}G_{\mathrm{Fe}: \mathrm{Va}}=\mathrm{GFCCFE}, G_{\mathrm{Mn}: \mathrm{Va}}=\mathrm{GFCCMN}, G_{\mathrm{Al}: \mathrm{Va}}=\mathrm{GHSERAL} \\
L_{\mathrm{Fe}, \mathrm{Al}: \mathrm{Va}}=-105855.20+30.65 T+(29016.76-4.91 T)\left(y_{\mathrm{Al}}^{\prime}-y_{\mathrm{Fe}}^{\prime}\right)+(32200-17 T)\left(y_{\mathrm{Al}}^{\prime}-y_{\mathrm{Fe}}^{\prime}\right)^{2[10]} \\
L_{\mathrm{Fe}, \mathrm{Mn}: \mathrm{Va}}=-7762+3.87 T-259\left(y_{\mathrm{Fe}}^{\prime}-y_{\mathrm{Mn}}^{\prime}\right)^{[27]} \\
L_{\mathrm{Al}, \mathrm{Mn}: \mathrm{Va}}=-69938+27.18 T+8248.9\left(y_{\mathrm{Al}}^{\prime}-y_{\mathrm{Mn}}^{\prime}\right)^{[38]} \\
L_{\mathrm{Fe}, \mathrm{Al}, \mathrm{Mn}: \mathrm{Va}}=-25104 y_{\mathrm{Mn}}^{\prime} \\
T_{\mathrm{cFe}: \mathrm{Va}}=-201, T_{\mathrm{cMn}: \mathrm{Va}}=-1620, \beta_{\mathrm{Fe}: \mathrm{Va}}=-2.1, \beta_{\mathrm{Mn}: \mathrm{Va}}=-1.86^{[27]} \\
T_{\mathrm{cFe}, \mathrm{Mn}: \mathrm{Va}}=-2282-2068\left(y_{\mathrm{Fe}}^{\prime}-y_{\mathrm{Mn}}^{\prime}\right)^{[27]}\end{array}$ \\
\hline bcc_A2 & $(\mathrm{Fe}, \mathrm{Mn}, \mathrm{Al})_{1}(\mathrm{Va})_{3}$ & $\begin{array}{l}G_{\mathrm{Fe}: \mathrm{Va}}=\mathrm{GHSERFE}, G_{\mathrm{Mn}: \mathrm{Va}}=\mathrm{GBCCMN}, G_{\mathrm{Al}: \mathrm{Va}}=\mathrm{GBCCAL} \\
L_{\mathrm{Fe}, \mathrm{Al}: \mathrm{Va}}=-123044+32 T++2945.20\left(y_{\mathrm{Al}}^{\prime}-y_{\mathrm{Fe}}^{\prime}\right)-3347.20\left(y_{\mathrm{Al}}^{\prime}-y_{\mathrm{Fe}}^{\prime}\right)^{2}[10] \\
L_{\mathrm{Fe}, \mathrm{Mn}: \mathrm{Va}}=-2759+1.24 T^{[27]} \\
L_{\mathrm{Al}, \mathrm{Mn}: \mathrm{Va}}=-111336.2+44.48 T+(-68691.8+44.53 T)\left(y_{\mathrm{Al}}^{\prime}-y_{\mathrm{Mn}}^{\prime}\right)^{[38]} \\
L_{\mathrm{Fe}, \mathrm{Al}, \mathrm{Mn}: \mathrm{Va}}=4184 \\
T_{\mathrm{cFe}: \mathrm{Va}}=1043, T_{\mathrm{cMn}: \mathrm{Va}}=-580, \beta_{\mathrm{Fe}: \mathrm{Va}}=2.22, \beta_{\mathrm{Mn}: \mathrm{Va}}=-0.27^{[27]} \\
T_{\mathrm{cFe}, \mathrm{Mn}: \mathrm{Va}}=123^{[27]} \\
T_{\mathrm{cFe}, \mathrm{Al}: \mathrm{Va}}=-438+1720\left(y_{\mathrm{Fe}}^{\prime}-y_{\mathrm{Al}}^{\prime}\right)^{[32]}\end{array}$ \\
\hline bcc_B2 & $\begin{array}{l}(\mathrm{Fe}, \mathrm{Mn}, \mathrm{Al})_{0.5} \\
(\mathrm{Fe}, \mathrm{Mn}, \mathrm{Al})_{0.5}(\mathrm{Va})_{3}\end{array}$ & $\begin{array}{l}G_{\mathrm{Fe}: \mathrm{Fe}: \mathrm{Va}}=G_{\mathrm{Al}: \mathrm{Al}: \mathrm{Va}}=G_{\mathrm{Mn}: \mathrm{Mn}: \mathrm{Va}}=G_{\mathrm{Fe}: \mathrm{Mn}: \mathrm{Va}}=G_{\mathrm{Mn}: \mathrm{Fe}: \mathrm{Va}}=0 \\
(\rightarrow) G_{\mathrm{Fe}: \mathrm{Al}: \mathrm{Va}}=G_{\mathrm{Al}: \mathrm{Fe}: \mathrm{Va}}=-16092-2.95 T{ }^{[10]} G_{\mathrm{Al}: \mathrm{Mn}: \mathrm{Va}}=G_{\mathrm{Mn}: \mathrm{Al}: \mathrm{Va}}=-10250 \\
T_{\mathrm{cFe}: \mathrm{Al}: \mathrm{Va}}=T_{\mathrm{cAl}: \mathrm{Fe}: \mathrm{Va}}=-250,{ }^{[32]} \beta_{\mathrm{Fe}: \mathrm{Al}: \mathrm{Va}}=\beta_{\mathrm{Al}: \mathrm{Fe}: \mathrm{Va}}=-2.72^{[32]} \\
(\rightarrow) L_{\mathrm{Fe}, \mathrm{Al}: \mathrm{Al}: \mathrm{Va}}=1665.37-4 T-524\left(y_{\mathrm{Al}}^{\prime}-y_{\mathrm{Fe}}^{\prime}\right)-1560\left(y_{\mathrm{Al}}^{\prime}-y_{\mathrm{Fe}}^{\prime}\right)^{2[32]} \\
(\rightarrow) L_{\mathrm{Al}: \mathrm{Fe}, \mathrm{Al}: \mathrm{Va}}=1665.37-4 T-524\left(y_{\mathrm{Al}}^{\prime \prime}-y_{\mathrm{Fe}}^{\prime \prime}\right)-1560\left(y_{\mathrm{Al}}^{\prime \prime}-y_{\mathrm{Fe}}^{\prime \prime}\right)^{2[32]} \\
(\rightarrow) L_{\mathrm{Fe}, \mathrm{Al}: \mathrm{Fe}: \mathrm{Va}}=-5346-1.6 T-524\left(y_{\mathrm{Al}}^{\prime}-y_{\mathrm{Fe}}^{\prime}\right)-1560\left(y_{\mathrm{Al}}^{\prime}-y_{\mathrm{Fe}}^{\prime}\right)^{2[32]} \\
(\rightarrow) L_{\mathrm{Fe}: \mathrm{Fe}, \mathrm{Al}: \mathrm{Va}}=-5346-1.6 T-524\left(y_{\mathrm{Al}}^{\prime \prime}-y_{\mathrm{Fe}}^{\prime \prime}\right)-1560\left(y_{\mathrm{Al}}^{\prime \prime}-y_{\mathrm{Fe}}^{\prime \prime}\right)^{2[32]} \\
T_{\mathrm{cFe}, \mathrm{Al} \mathrm{Al}: \mathrm{Va}}=T_{\mathrm{cFe}, \mathrm{Al}: \mathrm{Fe}: \mathrm{Va}}=T_{\mathrm{cFe}: \mathrm{Fe}, \mathrm{Al}: \mathrm{Va}}=T_{\mathrm{cAl}: \mathrm{Fe}, \mathrm{Al}: \mathrm{Va}}=250^{[32]} \\
\beta_{\mathrm{Fe}, \mathrm{Al}: \mathrm{Al}: \mathrm{Va}}=\beta_{\mathrm{Fe}, \mathrm{Al}: \mathrm{Fe}: \mathrm{Va}}=-0.6-1.6\left(y_{\mathrm{Al}}^{\prime}-y_{\mathrm{Fe}}^{\prime}\right)+0.4\left(y_{\mathrm{Al}}^{\prime}-y_{\mathrm{Fe}}^{\prime}\right)^{2[32]} \\
\beta_{\mathrm{Fe}: \mathrm{Fe}, \mathrm{Al}: \mathrm{Va}}=\beta_{\mathrm{All}: \mathrm{Fe}, \mathrm{Al}: \mathrm{Va}}=-0.6-1.6\left(y_{\mathrm{Al}}^{\prime \prime}-y_{\mathrm{Fe}}^{\prime \prime}\right)+0.4\left(y_{\mathrm{Al}}^{\prime \prime}-y_{\mathrm{Fe}}^{\prime \prime}\right)^{2[32]} \\
L_{\mathrm{Fe}, \mathrm{Al}: \mathrm{Fe}, \mathrm{Al}: \mathrm{Va}}=-8400-1.8 T^{[10]} \\
L_{\mathrm{Fe}, \mathrm{Al}: \mathrm{Mn}: \mathrm{Va}}=L_{\mathrm{Mn}: \mathrm{Fe}, \mathrm{Al}: \mathrm{Va}}=-4184\end{array}$ \\
\hline cbcc & $(\mathrm{Fe}, \mathrm{Mn}, \mathrm{Al})_{1}(\mathrm{Va})_{1}$ & $\begin{array}{l}G_{\mathrm{Fe}: \mathrm{Va}}=\text { GHSERFE }+4745,{ }^{[27]} G_{\mathrm{Mn}: \mathrm{Va}}=\text { GHSERMN }, G_{\mathrm{Al}: \mathrm{Va}}=\mathrm{GCBCCAL} \\
L_{\mathrm{Fe}, \mathrm{Mn}: \mathrm{Va}}=-10184^{[27]} \\
L_{\mathrm{Al}, \mathrm{Mn}: \mathrm{Va}}=-63000+21 T^{[38]} \\
(\rightarrow) T_{\mathrm{cMn}: \mathrm{Va}}=-285, \beta_{\mathrm{Mn}: \mathrm{Va}}=-0.66^{[29]}\end{array}$ \\
\hline cub & $(\mathrm{Fe}, \mathrm{Mn}, \mathrm{Al})_{1}(\mathrm{Va})_{1}$ & $\begin{array}{l}G_{\mathrm{Fe}: \mathrm{Va}}=\text { GHSERFE }+3745,{ }^{[27]} G_{\mathrm{Mn}: \mathrm{Va}}=\text { GCUBMN }, G_{\mathrm{Al}: \mathrm{Va}}=\mathrm{GCUBAL} \\
L_{\mathrm{Fe}, \mathrm{Al}: \mathrm{Va}}=-129704+41.84 T^{[10]} \\
L_{\mathrm{Fe}, \mathrm{Mn}: \mathrm{Va}}=-11518+2.82 T^{[27]} \\
L_{\mathrm{Al}, \mathrm{Mn}: \mathrm{Va}}=-118512+52.74 T-3174.9\left(y_{\mathrm{Al}}^{\prime}-y_{\mathrm{Mn}}^{\prime}\right)^{[38]} \\
L_{\mathrm{Fe}, \mathrm{Mn}, \mathrm{Al}: \mathrm{Va}}=-46024 y_{\mathrm{Fe}}^{\prime}+(-30815.2-20.92 T) y_{\mathrm{Al}}^{\prime}\end{array}$ \\
\hline hcp & $(\mathrm{Fe}, \mathrm{Mn}, \mathrm{Al})_{1}(\mathrm{Va})_{0.5}$ & $\begin{array}{l}G_{\mathrm{Fe}: \mathrm{Va}}=\mathrm{GHCPFE}, G_{\mathrm{Mn}: \mathrm{Va}}=\mathrm{GHCPMN}, G_{\mathrm{Al}: \mathrm{Va}}=\mathrm{GHCPAL} \\
L_{\mathrm{Fe}, \mathrm{Al}: \mathrm{Va}}=-40000^{[21]} \\
L_{\mathrm{Fe}, \mathrm{Mn}: \mathrm{Va}}=-5748+3.87 T+273\left(y_{\mathrm{Fe}}^{\prime}-y_{\mathrm{Mn}}^{\prime}\right)^{[20]} \\
(\rightarrow) L_{\mathrm{Al}, \mathrm{Mn}: \mathrm{Va}}=-95264.1+33.10 T+(-56880.6+43.14 T)\left(y_{\mathrm{Al}}^{\prime}-y_{\mathrm{Mn}}^{\prime}\right)+14430.2\left(y_{\mathrm{Al}}^{\prime}-y_{\mathrm{Mn}}^{\prime}\right)^{2[38]} \\
(\rightarrow) T_{\mathrm{cMn}: \mathrm{Va}}=-1620, \beta_{\mathrm{Mn}: \mathrm{Va}}=-1.86^{[27]}\end{array}$ \\
\hline $\mathrm{Al}_{8} \mathrm{Fe}_{5}$ & $(\mathrm{Al}, \mathrm{Fe})_{8}(\mathrm{Al}, \mathrm{Fe})_{5}$ & $\begin{array}{l}G_{\mathrm{Al}: \mathrm{Al}}=13 \mathrm{GBCCAL},{ }^{[32]} G_{\mathrm{Fe}: \mathrm{Fe}}=13 \mathrm{GHSERFE}+13000^{[32]} \\
G_{\mathrm{Al}: \mathrm{Fe}}=8 \mathrm{GBCCAL}+5 \mathrm{GHSERFE}-384500+30 T^{[10]} \\
G_{\mathrm{Fe}: \mathrm{Al}}=8 \mathrm{GHSERFE}+5 \mathrm{GBCCAL}+200000+30 T^{[10]} \\
L_{\mathrm{Al}: \mathrm{Al}, \mathrm{Fe}}=-133888,{ }^{[10]} L_{\mathrm{Al}, \mathrm{Fe}: \mathrm{Fe}}=-174000^{[32]}\end{array}$ \\
\hline $\mathrm{Al}_{13} \mathrm{Fe}_{4}$ & $(\mathrm{Al})_{32}(\mathrm{Fe})_{12}(\mathrm{Al}, \mathrm{Va})_{7}$ & $\begin{array}{l}G_{\mathrm{Al}: \mathrm{Fe}: \mathrm{Al}}=39 \text { GHSERAL }+12 \text { GHSERFE }-1564680+377 T^{[32]} \\
G_{\mathrm{Al}: \mathrm{Fe}: \mathrm{Va}}=32 \mathrm{GHSERAL}+12 \mathrm{GHSERFE}-1433100+377 T^{[32]}\end{array}$ \\
\hline $\mathrm{Al}_{8} \mathrm{Mn}_{5}$ & $\mathrm{Al}_{12} \mathrm{Mn}_{5}(\mathrm{Al}, \mathrm{Mn})_{9}$ & $G_{\mathrm{Al}: \mathrm{Mn}: \mathrm{Al}}=21 \mathrm{GHSERAL}+5 \mathrm{GHSERMN}-199851.6-60.66 T^{[38]}$ \\
\hline
\end{tabular}


Table 4 continued

\begin{tabular}{|c|c|c|}
\hline Phase & Model & Parameter \\
\hline $\mathrm{HTAl}_{11} \mathrm{Mn}_{4}$ & $(\mathrm{Al}, \mathrm{Mn})_{29} \mathrm{Mn}_{10}$ & $\begin{array}{l}G_{\mathrm{Al}: \mathrm{Mn}: \mathrm{Mn}}=12 \text { GHSERAL }+14 \text { GHSERMN }-791382.8+289.48 T^{[38]} \\
L_{\mathrm{Al}: \mathrm{Mn}: \mathrm{Al}, \mathrm{Mn}}=-522189.2+244.35 T+38014.6\left(y_{\mathrm{Al}}^{\prime \prime \prime}-y_{\mathrm{Mn}}^{\prime \prime \prime}\right)^{[38]} \\
G_{\mathrm{Al}: \mathrm{Mn}}=29 \mathrm{GHSERAL}+10 \mathrm{GHSERMN}-744942.9+130.36 T^{[38]} \\
G_{\mathrm{Mn}: \mathrm{Mn}}=39 \text { GHSERMN }+195000^{[38]} \\
L_{\mathrm{Al}, \mathrm{Mn}: \mathrm{Mn}}=-664033.5^{[38]}\end{array}$ \\
\hline $\mathrm{Al}_{2} \mathrm{Fe}$ & & $G=2 \mathrm{GHSERAL}+\mathrm{GHSERFE}-94850+13.42 T^{[32]}$ \\
\hline $\mathrm{Al}_{5} \mathrm{Fe}_{2}$ & & $G=5$ GHSERAL +2 GHSERFE $-217301+34.83 T^{[32]}$ \\
\hline $\mathrm{LTAl}_{11} \mathrm{Mn}_{4}$ & & $G=11 \mathrm{GHSERAL}+4 \mathrm{GHSERMN}-350550+97.90 T^{[38]}$ \\
\hline $\begin{array}{l}\lambda-\mathrm{Al}_{4} \mathrm{Mn} \\
\left(\mathrm{Al}_{461} \mathrm{Mn}_{107}\right)^{[38]}\end{array}$ & $(\rightarrow) \mathrm{Al}_{99} \mathrm{Mn}_{23} *$ & $G=99 \mathrm{GHSERAL}+23 \mathrm{GHSERMN}-2506648.6+835.55 T$ \\
\hline$\mu-\mathrm{Al}_{4} \mathrm{Mn}$ & & $G=4$ GHSERAL + GHSERMN $-109088.5+36.67 T^{[38]}$ \\
\hline $\mathrm{Al}_{6} \mathrm{Mn}$ & & $G=6$ GHSERAL + GHSERMN $-109090.8+35.22 T^{[38]}$ \\
\hline $\mathrm{Al}_{12} \mathrm{Mn}$ & & $G=12$ GHSERAL + GHSERMN $-110389.5+36.80 T^{[38]}$ \\
\hline
\end{tabular}

